\title{
The relationship between calcium metabolism, insulin- like growth factor-1 and pulse pressure in normotensive, normolipidaemic and non-diabetic patients
}

\author{
Sibel Ertek ${ }^{1}$, Arrigo Francesco Cicero², Gürbüz Erdoğan ${ }^{1}$
}

1Department of Endocrinology and Metabolic Diseases, Ufuk University Medical
Faculty, Ankara, Turkey
2Internal Medicine, Aging and Kidney Diseases Department, Atherosclerosis and
Metabolic Diseases Research Center, Bologna University, Bologna, Italy

Submitted: 26 April 2011

Accepted: 6 September 2011

Arch Med Sci 2011; 7, 5: 776-780

DOI: 10.5114/aoms.2011.25551

Copyright @ 2011 Termedia \& Banach

\author{
Corresponding author: \\ Sibel Ertek MD \\ Department of Endocrinology \\ and Metabolic Diseases \\ Ufuk University \\ Dr. Ridvan Ege Hospital \\ Mevlana Bulvari 86-88 \\ 06520 Balgat-Ankara, Turkey \\ Phone: +90312 20440 00/ \\ 4230 \\ Fax: +90 3122044055 \\ E-mail: sibelertek@yahoo.it
}

\begin{abstract}
Introduction: Recent evidence suggests an interaction between bone metabolism and blood pressure (BP) regulation. The aim of our study was to evaluate endocrinological and metabolic factors related to pulse pressure (PP) in normotensive, normolipidaemic, non-smoker subjects.

Material and methods: We consecutively enrolled 156 adults (37 males, 119 females) in summer 2009. The BP and body mass index (BMI) were recorded, and serum samples were taken for 25-hydroxy vitamin D (25-OHD), insulin-like growth factor-1 (IGF-1), growth hormone (GH), parathormone (PTH), calcium, albumin, phosphorus, glucose, triglyceride and cholesterol levels.

Results: In the postmenopausal group, PP was significantly associated with age and BMI, while in premenopausal patients it was inversely related to ionized calcium. In men, a reverse relationship was present between GH and IGF-1 levels and PP.

Conclusions: The PP was predicted by different parameters in different genders and these predictors change even in the same gender before and after menopause. Well-known predictors of PP such as age and BMI were more pronounced in postmenopausal women, but none of the groups showed a relationship between PP and 25-OHD or PTH.
\end{abstract}

Key words: vitamin D, blood pressure, insulin-like growth factor-1, calcium, body mass index.

\section{Introduction}

Abundant recent evidence suggests a complex interaction between bone metabolism and blood pressure regulation. A recent meta-analysis of ten observational studies and nine randomized controlled trials supports a relationship between high blood pressure and low vitamin D levels [1]. Parathyroid hormone (PTH) level is a known cardiovascular risk predictor in patients with chronic renal disease (CRD) [2], but studies about its effect on blood pressure in normal people are lacking.

The relationship of insulin-like growth factor-1 (IGF-1), growth hormone $(\mathrm{GH})$ and blood pressure is based on observations in patients with acromegaly and obstructive sleep apnoea syndrome [3, 4], but their interrelationship in normal subjects is not clear. Some studies suggest an 
interaction between IGF and vitamin D metabolism: active vitamin D (1,25-hydroxy-cholecalcipherol) may increase IGF-1 receptors and IGF-1 may augment renal 1- $\alpha$ hydroxylase expression, so increasing the active form of vitamin D [5]. Meanwhile, insulin-like growth factor binding proteins may interact with vitamin $\mathrm{D}$ receptors, inducing the expression of bone differentiation markers [6]. More recent studies also show a relationship between vitamin D and IGF-1 in metabolic syndrome patients [7].

There is also a strong association between serum 25-hydroxy vitamin D (25-OHD) levels and body mass index (BMI): obese people have lower 25-OHD levels than non-obese people [8]. Meanwhile, recently it was observed with the calcium-citrate clamping test that obese subjects have disturbed calcium homeostasis that is very similar to that related to compensatory mechanisms in patients with early renal insufficiency, showing a left-sided shift in the calcium-PTH curve, indicating a lower set point, related to the duration of obesity [9]. But still there is no practical application of this knowledge.

Pulse pressure (PP) is a non-invasively measured parameter related to cardiovascular risk, showing the difference between pulsatile stress of large artery stiffness during systole and steady-state stress of small vessel resistance during diastole [10]. The PP is calculated as the difference between systolic and diastolic blood pressures and normally it is $30-40 \mathrm{mmHg}$. Wider pulse pressure is a known risk factor associated with higher mortality and higher risk of myocardial infarction [11].

In this context, the aim of our study was to evaluate endocrinological and metabolic factors related to PP among genders in non-obese, normotensive, non-smoking, non-diabetic subjects. In particular, we aimed to test for any differences between premenopausal and postmenopausal women, and men, as regards calcium metabolismrelated parameters, IGF-1 and $\mathrm{GH}$, and their relationship with PP.

\section{Material and methods}

We enrolled 156 patients (37 men, 119 women; 41 postmenopausal and 78 premenopausal) between 19 years and 70 years of age, without hypertension, hyperlipidaemia, diabetes, peripheral or coronary arterial disease in June-September 2010. None of them was a smoker. All were normocalcaemic and normoalbuminaemic. Patients using vitamin or calcium preparations, or with renal or hepatic disease, were excluded. Ethical Committee approval was obtained from the local ethical committee. The study was explained in detail to each subject and informed consent was obtained from each person.

We recorded weight, height and blood pressure, measured by a sphygmomanometer (Erka, Perfect
Aneroid with auto valve Sphygmomanometer, with adult 'size 4' cuffs) and recorded in a sitting position from both arms supported at heart level. The measurements were repeated 3 times with minimum 10-min intervals, and the mean value was calculated. The PP was calculated as the difference between systolic (SBP) and diastolic blood pressures (DBP) [10]. The BMI was calculated as weight in kilograms divided by square of height in metres $\left(\mathrm{kg} / \mathrm{m}^{2}\right)$.

\section{Biochemical analysis}

Venous blood samples were obtained early in the morning for measuring 12-h fasting blood glucose, total cholesterol, high-density lipoprotein (HDL) cholesterol, low-density lipoprotein (LDL) cholesterol, triglyceride, calcium, phosphorus, ionized calcium, IGF-1, and PTH levels with lab kits of USA origin. The PTH levels were measured by electrochemiluminescence immunoassay (ECLIA, Cobas $^{\circledR}$ ) [12] using an Elecsys 2010 analyser (USA) with an inter-assay coefficient of variation below or equal to $6.5 \%$, and IGF-1 was measured by immunoradiometric assay (IRMA, Immunotech) [13] with an intra-assay coefficient of variation of below or equal to $6.3 \%$ and an inter-assay coefficient of variation of below or equal to $6.8 \%$. 25-OHD measurements were obtained by standard highperformance liquid chromatography (HPLC, Immündiagnostik) [14]. Growth hormone measurement was performed by the IRMA method (Immunotech with inter-assay coefficient of variation $\leq 14.0 \%)$. The other parameters were measured by a standardized automatic colorimetric method using the Cobas Integra 400 Analyzer (USA). Tests were done immediately, just after taking blood samples in the morning of the patient's visit to the ambulatory.

\section{Statistical analysis}

Patients were grouped as men, premenopausal women and postmenopausal women. After normality distribution testing by KolmogorovSmirnov test, and parameter log-normalization (when needed) the continuous variables of these three groups were compared by ANOVA test followed by post-hoc Tukey HSD test. Multiple regression analysis was performed to determine the parameters related to PP for each patient group separately. A $p$ value $<0.05$ was considered significant for all tests. All statistical calculations were executed by the program SPSS 15.0 (for Office).

\section{Results}

Among the 156 adults included in the study, women $(n=119)$ were grouped in pre- and postmenopausal groups; thus we had 3 groups to 
compare. The mean and standard deviation of measured parameters are summarized in Tables I and II.

We observed that age was significantly higher in postmenopausal patients than all other groups (men vs. postmenopausal women $p<0.05$, postvs. pre-menopausal groups $p<0.05$, men vs. premenopausal group $p<0.01$ ). Also, BMI of postmenopausal women was higher than that of premenopausals $(p<0.05)$. Premenopausal women had higher $\mathrm{GH}$ levels than men $(p<0.05)$ and postmenopausals $(p<0.01)$. Premenopausal women also had significantly lower triglyceride levels than others (premenopausal group vs. men, $p<0.05$; pre- vs. post-menopausal women, $p<0.01$ ) and HDL levels were higher in the premenopausal group than men $(p<0.01)$. Meanwhile premenopausal women also had lower SBP than the other two groups ( $p=0.001$ for both), lower DBP than men $(p<0.01)$ and postmenopausal women $(p<0.01)$, and also lower PP than men $(p<0.01)$ and postmenopausals $(p<0.01)$.

In the postmenopausal group, regression analysis showed a significant relationship between PP and age and BMI. In premenopausal patients, PP was inversely associated with ionized calcium. In men, IGF-1 and GH levels were inversely related to PP (Table III).

\section{Discussion}

Blood pressure control is important for cardiovascular risk reduction and usually studies related to pulse pressure and systemic blood pressure were carried out on hypertensive patients [15]. In this study, we evaluated the association of calcium metabolism-related factors, GH and IGF-1 with PP in normotensive subjects grouped as men, and post- and pre-menopausal women. Higher incidence of hypertension in postmenopausal women with low bone density is well known, but

Table I. Characteristics and biochemical parameters of the studied groups (mean \pm SD)

\begin{tabular}{|c|c|c|c|c|c|}
\hline Parameter & $\begin{array}{l}\text { All patients }{ }^{\dagger} \\
\quad(n=156)\end{array}$ & $\begin{array}{l}\text { Pre-menopausal } \\
\qquad(n=78)\end{array}$ & $\begin{array}{l}\text { Post-menopausal } \\
\qquad(n=41)\end{array}$ & $\begin{array}{c}\text { Men } \\
(n=37)\end{array}$ & $\begin{array}{l}\text { Values of } p \\
\left(\text { ANOVA) }{ }^{\star *}\right.\end{array}$ \\
\hline Age [years] & $41.9 \pm 1.3$ & $35.2 \pm 8.4$ & $51.6 \pm 4.7$ & $46.0 \pm 12.5$ & $0.001^{*}$ \\
\hline $\mathrm{BMI}\left[\mathrm{kg} / \mathrm{m}^{2}\right]$ & $27.62 \pm 5.3$ & $26.4 \pm 5.5$ & $29.2 \pm 3.8^{\star \star}$ & $28.4 \pm 6.5$ & $0.019^{*}$ \\
\hline $25 \mathrm{OHD}[\mu \mathrm{g} / \mathrm{l}])$ & $24.5 \pm 12.8$ & $22.22 \pm 12.54$ & $25.60 \pm 13.20$ & $27.40 \pm 11.83$ & NS \\
\hline Serum calcium $[\mathrm{mg} / \mathrm{dl}]$ & $9.43 \pm 0.42$ & $9.43 \pm 0.36$ & $9.34 \pm 0.47$ & $9.54 \pm 0.49$ & NS \\
\hline Albumin $[\mathrm{g} / \mathrm{dl}]$ & $4.45 \pm 0.34$ & $4.39 \pm 0.35$ & $4.32 \pm 0.18$ & $4.99 \pm 0.34$ & NS \\
\hline Ionized calcium [mmol/l] & $1.10 \pm 0.01$ & $1.11 \pm 0.03$ & $1.10 \pm 0.02$ & $1.09 \pm 0.03$ & NS \\
\hline Phosphorus [mg/dl] & $3.41 \pm 0.48$ & $3.43 \pm 0.44$ & $3.45 \pm 0.46$ & $3.31 \pm 0.54$ & NS \\
\hline PTH $[\mathrm{pg} / \mathrm{ml}]$ & $51.18 \pm 20.6$ & $52.00 \pm 19.82$ & $53.32 \pm 24.32$ & $47.40 \pm 18.47$ & NS \\
\hline $\mathrm{GH}[\mathrm{ng} / \mathrm{ml}]$ & $2.59 \pm 3.96$ & $3.95 \pm 4.98^{\star *}$ & $1.16 \pm 0.74$ & $1.62 \pm 2.98$ & $0.001^{*}$ \\
\hline IGF-1 [ng/ml] & $183.69 \pm 60.22$ & $201.44 \pm 49.03^{* *}$ & $153.59 \pm 49.24$ & $174.07 \pm 80.36$ & $0.001^{*}$ \\
\hline
\end{tabular}

BMI - body mass index, 25OHD - 25-OH vitamin D, PTH - parathormone, GH - growth hormone, IGF-1 - insulin-like growth hormone-1, NS - statistically not significant, ${ }^{*} p<0.05$ accepted as statistically significant, ${ }^{\dagger}$ all parameters are given as mean \pm standard deviation, ${ }^{* *}$ see the text for significant differences between three groups (revealed by post-hoc Tukey HSD test), normal ranges of hormone levels are: PTH 11-54 pg/ml, 25OHD > $20 \mu \mathrm{g} / \mathrm{l}, \mathrm{GFF}-190-300 \mathrm{ng} / \mathrm{ml}, \mathrm{GH}<5 \mathrm{ng} / \mathrm{ml}$

Table II. Blood pressure and lipid parameters of the studied groups (mean \pm SD)

\begin{tabular}{|lccccc|}
\hline Parameter & $\begin{array}{c}\text { All patients } \\
(n=156)\end{array}$ & $\begin{array}{c}\text { Pre-menopausal } \\
(n=78)\end{array}$ & $\begin{array}{c}\text { Post-menopausal } \\
(n=41)\end{array}$ & $\begin{array}{c}\text { Men } \\
(n=37)\end{array}$ & $\begin{array}{c}\text { Values of } p \\
(\text { ANOVA })^{\star *}\end{array}$ \\
\hline TG $[\mathrm{mg} / \mathrm{dl}]$ & $116.56 \pm 46.77$ & $101.03 \pm 40.17^{\star *}$ & $140.63 \pm 55.61$ & $123.83 \pm 37.92$ & $0.001^{*}$ \\
\hline $\mathrm{LDL}-\mathrm{C}[\mathrm{mg} / \mathrm{dl}]$ & $121.01 \pm 27.10$ & $118.05 \pm 28.77$ & $127.76 \pm 21.73$ & $119.37 \pm 29.18$ & $\mathrm{NS}$ \\
\hline $\mathrm{HDL}-\mathrm{C}[\mathrm{mg} / \mathrm{dl}]$ & $54.59 \pm 15.14$ & $58.67 \pm 14.79^{* *}$ & $52.68 \pm 12.35$ & $48.48 \pm 16.66$ & $0.002^{*}$ \\
\hline SBP $[\mathrm{mmHg}]$ & $123.3 \pm 12.8$ & $117.6 \pm 10.2^{* *}$ & $129.4 \pm 13.5$ & $129.76 \pm 11.2$ & $0.001^{*}$ \\
\hline DBP $[\mathrm{mmHg}]$ & $77.9 \pm 9.5$ & $75.0 \pm 9.1^{* *}$ & $81.0 \pm 9.4$ & $81.1 \pm 8.7$ & $0.001^{*}$ \\
\hline PP $[\mathrm{mmHg}]$ & $45.4 \pm 10.4$ & $42.39 \pm 8.4^{* *}$ & $48.3 \pm 10.7$ & $48.85 \pm 12.7$ & $0.001^{*}$ \\
\hline
\end{tabular}

TG - triglycerides, LDL-C - low-density lipoprotein cholesterol, HDL-C-high-density lipoprotein cholesterol, SBP-systolic blood pressure, $D B P$ - diastolic blood pressure, NS - statistically not significant, ${ }^{*} p<0.05$ accepted as statistically significant, tall parameters are given as mean \pm standard deviation, ${ }^{* *}$ see the text for significant differences between three groups (revealed by post-hoc Tukey HSD test) 
Table III. Factors that were significantly related to pulse pressure in regression analysis for each group

\begin{tabular}{|c|c|c|c|c|}
\hline Factor & Value of $\beta$ & Value of $p$ & $95 \% \mathrm{Cl}$ upper bound & $95 \% \mathrm{Cl}$ lower bound \\
\hline \multicolumn{5}{|c|}{ Postmenopausal women } \\
\hline Age & 0.228 & 0.024 & 0.03 & 0.425 \\
\hline BMI & 0.390 & 0.035 & 0.028 & 0.751 \\
\hline \multicolumn{5}{|c|}{ Premenopausal women } \\
\hline Ionized calcium & -85.568 & 0.008 & -148.21 & -23.115 \\
\hline \multicolumn{5}{|l|}{ Men } \\
\hline IGF-1 & -0.081 & 0.004 & -0.134 & -0.028 \\
\hline$\overline{\mathrm{GH}}$ & -1.615 & 0.036 & -3.112 & -0.118 \\
\hline
\end{tabular}

the effect of calcium metabolism-related factors on blood pressure is not clear [16]. Vitamin D may have a role in the association between atherosclerosis and osteoporosis in this group of patients, but the role of vitamin $D$ in blood pressure levels in normotensive subjects is not yet well known [17].

The PP is a marker of stiffening of large arteries and is a well-known independent predictor of cardiovascular risk [18-21]. Since stiffening of arteries is related to age, in our study, in fact, we observed a direct relationship between age and PP in the postmenopausal group that has higher mean age than both other considered groups. The same group also had the highest BMI, which was also consistent with literature data [22].

Since the pulsatile secretion of $\mathrm{GH}$ is more important for evaluation, we measured IGF-1 levels together with $\mathrm{GH}$. Similarly, we recorded ionized calcium levels of patients to avoid factors affecting total serum calcium levels. Higher GH and IGF-1 levels in premenopausal women may parallel their lower mean age as expected, but the reverse relationship between IGF-1 and GH levels and PP in men should be confirmed with physiological studies. A negative relationship between adiposity and $\mathrm{GH}$ $[23,24]$ may be a theoretical explanation, but in our study men did not show a significant difference from others regarding BMI. These findings on relations between blood pressure and GH and IGF-1 levels are parallel with known observations in the medical literature as observed in special patient groups with acromegaly or sleep apnoea [3,4]. Postmenopausal women with higher BMI did not reveal any relationship between PP and GH or IGF-1, probably because some other factors (such as age and BMI found in this study) dominate.

lonized calcium and pulse pressure were inversely related in premenopausal patients. There are many studies related to calcium metabolism and PP in chronic renal failure patients but not in the healthy population $[25,26]$. This younger and relatively thinner group revealed lower systolic and diastolic blood pressure and consequently lower pulse pressure values than the other two groups. In the study of Fraser et al., albumin adjusted calcium levels were associated with higher systolic blood pressure in three National Health and Nutrition Examination Survey (NHANES) cycles [27].

While the above-cited meta-analysis of Feneis et al. [1] supported a significant association between hypertension and low serum vitamin D, another recent meta-analysis of 3 cohorts did not find a significant association between vitamin $\mathrm{D}$ and incident hypertension [28]. Thus, the medical literature still has no consensus about the relationship between calcium homeostasis and cardiovascular outcomes.

Our study also did not reveal a relationship between PP and 25-OHD or PTH in pre- and postmenopausal women or in men. In the study of Chan et al. carried out on elderly men, PTH was associated with blood pressure but serum 25-OHD levels were not found to be related [29]. Similarly, the study of Hjelmesath et al. also revealed an association of metabolic syndrome and PTH levels but not serum 25-OHD levels in morbidly obese people [30].

Despite the fact that the study was executed in summer, the mean 25-OHD level of patients in any group is slightly higher than the lower limit (i.e. $20 \mu \mathrm{g} / \mathrm{l}$ ) suggested by the American Endocrine Society [31]. Its role in the study results was unpredictable.

Although to the best of our knowledge in the medical literature there is no other study comparing healthy men and pre- and post-menopausal women regarding pulse pressure and calcium metabolism related parameters, our study still has some restrictions. Firstly, the blood pressure measurements were not made by ambulatory 24-h measurements, which may give more valuable and detailed information including nocturnal changes. Secondly, although we compared three groups, the relatively low number of males may restrict the evaluation of gender differences. And since it is a cross-sectional study, we cannot speculate on 
future results of vitamin D status, or effects of treatment. Meanwhile, $\mathrm{GH}$ is affected by many factors and its spot measurement may not suggest specific pathology unless it exceeds a threshold, but we also measured and included IGF-1 to avoid misleading results due to fluctuations in $\mathrm{GH}$ levels.

In conclusion, in our normotensive, non-smoker, non-diabetic, normolipidaemic study group, PP was related to age and BMI in postmenopausal women, inversely related to ionized calcium in premenopausal women and also inversely related to IGF-1 and $\mathrm{GH}$ in men, and none of the groups showed a relationship between PP and 25-OHD or PTH. That suggests changing dynamics of the relationship between PP and GH, IGF-1 and calcium metabolism markers according to gender, age, BMI and also menopausal status.

\section{References}

1. Feneis JF, Arora RR. Role of vitamin D in blood pressure homeostasis. Am J Ther 2010; 17: e221-9.

2. Bhuriya R, Li S, Chen SC, McCullough PA, Bakris GL. Plasma parathyroid hormone level and prevalent cardiovascular disease in CKD stages 3 and 4: an analysis from the Kidney Early Evaluation Program (KEEP). Am J Kidney Dis 2009; 53 (Suppl 4): S3-10.

3. Lanfranco F, Motta G, Minetto MA, Ghigo E, Maccario M. Growth hormone/insulin-like growth factor-I axis in obstructive sleep apnea syndrome: an update. J Endocrinol Invest 2010; 33: 192-6.

4. Colao A, Terzolo M, Bondanelli M, et al. GH and IGF-I excess control contributes to blood pressure control: results of an observational, retrospective, multicentre study in 105 hypertensive acromegalic patients on hypertensive treatment. Clin Endocrinol 2008; 69: 613-20.

5. Gomez JM. The role of insulin like growth factor-1 components in the regulation of vitamin D. Curr Pharm Biotechnol 2006; 7: 125-32.

6. Schedlich LJ, Muthukaruppan A, O'Han MK, Baxter RC. Insulin-like growth factor binding protein-5 interacts with the vitamin D receptor and modulates the vitamin D response in osteoblasts. Mol Endocrinol 2007; 21: 2378-90.

7. Hyppönen E, Boucher BJ, Berry DJ, Power C. 25-hydroxy vitamin D, IGF-1, and metabolic syndrome at 45 years of age: a cross-sectional study in the 1958 British Birth Cohort. Diabetes 2008; 57: 298-305

8. Jorde R, Sneve M, Emaus N, Figenschau Y, Grimnes G. Cross-sectional and longitudinal relation between serum 25-hydroxyvitamin D and body mass index: the Troms? study. Eur J Nutr 2010; 49: 401-7.

9. Hultin H, Edfeldt K, Sundbom M, Hellman P. Left-shifted relation between calcium and parathyroid hormone in obesity. J Clin Endocrinol Metab 2010; 95: 3973-81.

10. Franklin SS. Cardiovascular risks related to increased diastolic, systolic and pulse pressure. An epidemiologist's point of view. Pathol Biol 1999; 47: 594-603.

11. Millar AJ, Lever AF. Implications of pulse pressure as a predictor of cardiac risk in patients with hypertension. Hypertension 2000; 36: 907-11.

12. Endres DB, VillanuevaR, Sharp CF Jr, Singer FR. Measurement of parathyroid hormone. Endocrinol Metab Clin North Am 1989; 18: 611-29.
13. Clemmons DR. Commercial assays available for insulinlike growth factor 1 and their use in diagnosing growth hormone deficiency. Horm Res 2001; 55 Suppl 2: 73-9.

14. Hollis BW. The determination of circulating 25-hyrdoxy vitamin D: no esy task. J Clin Endocrinol Metab 2004; 89: 3149-51.

15. Sethi A, Arora RR. Ambulatory blood pressure as a predictor of cardiovascular risk. Arch Med Sci 2009; 5: 3-9.

16. Yazici S, Yazici M, Korkmaz U, et al. Relationship between blood pressure levels and bone mineral density in postmenopausal Turkish women. Arch Med Sci 2011; 7: 264-70.

17. Stojanovic Ol, Lazovic M, Vuceljic M. Association between atherosclerosis and osteoporosis, the role of vitamin D. Arch Med Sci 2011; 7: 179-88.

18. Blacher J, Staessen JA, Girerd X, et al. Pulse pressure not mean pressure determines cardiovascular risk in older hypertensive patients. Arch Intern Med 2004; 160: 1085-9.

19. Franklin SS, Khan SA, Wong ND, Larson MG, Levy D. Is pulse pressure useful in predicting risk for coronary heart disease? The Framingham heart study. Circulation 1999; 100: $354-60$

20. Benetos A. Pulse pressure and cardiometabolic risk. J Hypertens Suppl 1999; 17: S21-4.

21. Pede S, Lombardo M. Cardiovascular risk stratification. Systolic, diastolic or pulse pressure? Ital Heart J Suppl 2001; 2: 356-8.

22. Kwagyan J, Tabe CE, Xu S, Maqbool AR, Gordeuk VR, Randall OS. The impact of body mass index on pulse pressure in obesity. J Hypertens 2005; 23: 619-24.

23. Rasmussen $M$, Hvidberg A, Juul $A$, et al. Massive weight loss restores 24-hour growth hormone release profiles and serum insulin-like growth factor-1 levels in obese subjects. J Clin Endocrinol Metab 1995; 80: 1407-15.

24. Ciuoli C, Brusco L, Di Cosmo L, et al. Serum GH and IGF1 levels in obese patients treated with bariatric surgery. Nutr Ther Metab 2010; 28: 95-9.

25. Guérin AP, London GM, Marchais SJ, Metivier F. Arterial stiffening and vascular calcifications in end-stage renal disease. Nephrol Dial Transplant 2000; 15: 1014-21.

26. Inrig JK, Patel UD, Toto RD, et al. Decreased pulse pressure during hemodialysis is associated with improved 6-month outcomes. Kidney Int 2009; 76: 1098-107.

27. Fraser A, Williams D, Lawlor DA. Associations of serum 25-hydroxy vitamin D, parathormone, and calcium with cardiovascular risk factors: analysis of three NHANES cycles (2001-2006). PLoS One 2010; 5: e13882.

28. Pittas AG, Chung M, Trikalinos T, et al. Vitamin D and cardiometabolic outcomes. Ann Intern Med 2010; 152: 307-14.

29. Chan R, Chan D, Woo J, et al. Serum 25-hydroxy vitamin D and parathyroid hormone levels in relation to blood pressure in a cross sectional study in older Chinese men. J Hum Hypertens 2011 [Epub ahead of print].

30. Hjelmesæth J, Hofs $\varnothing$ D, Aasheim ET, et al. Parathyroid hormone but not vitamin D is associated with metabolic syndrome in morbidly obese women and men: a cross sectional study. Cardiovasc Diabetol 2009; 8: 7.

31. Ross AC, Mannson JE, Abrams SA, et al. The 2011 report on dietary reference intakes for calcium and vitamin D from the Institute of medicine: what clinicians need to know. J Clin Endocrinol Metab 2011; 96: 53-8. 